\title{
Digital pathology in academia: Implementation and impact
}

\author{
Yava Jones-Hall discusses the promise and pain points of setting up a digital pathology program at a new \\ academic institution.
}

Yava Jones-Hall

T he vision of a cohort of pathologists is often the impetus for a digital pathology (DP) initiative, but the challenges that must be to overcome at the outset - and the throughout the life of the DP program - may stymie that initial excitement. In my opinion, there are 3 top challenges: the need to shift some pathologists' mindsets about the usefulness and advantages of DP when compared to only using a light microscope; the significant time and financial investment required to start and maintain a DP program; and last, but certainly not least, the minefield comprised of academic restrictions and requirements and the risks and liabilities associated with a virtual platform.

The three issues listed above have, one by one or collectively, significantly slowed or entirely halted the implementation of DP initiatives at many institutions. In fact, implementation of a DP workflow would likely still be moving at a stilted pace in many institutions were if not for the imperative to find alternative (remote) ways to educate and work that was imposed during the COVID-19 pandemic. The COVID-19 pandemic essentially forced acceptance of switching to a digital platform in many arenas, including pathology education, diagnostics, and research, in order to continue to deliver material safely. Many institutions have subsequently adopted the DP platform; however, many are unfortunately addressing the 3 issues mentioned above retroactively-not ideal, but unavoidable.

For this article, we'll presume these 3 key elements are tackled from the beginning and cover some of the more salient considerations of each. Notably, the Digital Pathology Association (DPA) recently provided a practical guide that highlights some of the requirements of using whole slide image (WSI) in pathology, as well as a white paper with helpful computational pathology definitions and suggested best practices $^{1,2}$.

$\begin{aligned} & \text { Table } 1 \text { | Example of configuration recommendations that would facilitate the use of DP } \\
& \text { by individual PC computer system users }\end{aligned}$
\begin{tabular}{ll} 
HARDWARE/SOFTWARE & RECOMMENDATION \\
\hline Hard Drive & 256GB or greater \\
\hline Memory & $16 \mathrm{~GB}-32 \mathrm{~GB}$ of RAM \\
\hline Operating System & Windows 10 Professional - Current version \\
\hline Processor & Intel 10th generation i7 \\
\hline Memory & 8 Gb of RAM minimum \\
\hline Processor & Intel 8th Generation i5 \\
\hline Monitor size & $13^{\prime \prime}-15^{\prime \prime}$ \\
\hline Internet Speed / Bandwidth & at least 10 Mbps is preferred \\
\hline & Office Suite and Anti-Virus Protection
\end{tabular}

\section{Information Technology can help guide your way}

The seriousness of the potential risks and the complications of setting up a digital platform underscores the need for information technology (IT) to be involved at the very conception of a DP integration plan. Your IT (and risk management) teams will help ensure all purchases and plans are in alignment with your organization's requirements and infrastructure and can also assist with the vetting of products from an IT perspective-potentially saving one from making an expensive purchase that will not integrate well within your environment.

However, pathology and IT teams inherently speak different "languages" and are usually trying to achieve drastically different goals, which can make finding common ground and understanding challenging. Nevertheless, they must both persist. Many of the institutional requirements and regulations are under the purview of the IT department and must be met and adhered to in order to implement any computer-based platform-especially if students will be involved.

Often, distributors of both hardware and software for DP focus primarily on the science and less so on institutional IT regulations and IT security. IT security assesses and controls the threats and liabilities that may be associated with the electronic transmission of potentially sensitive and/or confidential material-e.g. digital slides, privileged information that may be contained in the distribution lists that receive the images, or the metadata that may be encoded in the digital file. To use the acquired and stored data, the computers in the system and the storage servers must communicate through a network, which often needs frequent repairs to address security vulnerabilities or upgrades. Thus, all vendors should be made aware of any scheduling requirements for repairs and upgrades to ensure compliance and continuity of service during maintenance.

Other details that should be determined prior to purchasing products can include: how the data will be managed and stored; what the impact on the DP service will be if the data were to be lost, damaged or (temporarily or permanently) be inaccessible; and the size of storage that will be needed during set up and long-term. To determine the size of storage (and therefore the cost), it is recommended to extrapolate the need of one year out to 5 years (allowing for an excess of at least $10 \%$ unfilled space to be reserved for IT maintenance operations) and budget accordingly. Table 1 provides 
an example of the individual PC computer requirements needed to support basic WSI viewing and slide analytics (Table 1 ).

\section{Time and money: The two biggest budgets}

Digital pathology often represents a new(er) area of research at an institution and is an overall expensive undertaking; as such, this is not an endeavor for the faint of heart or resolve. As mentioned above, both the hardware and software must be thoroughly investigated and the IT risks managed. Just as important as the guidance provided by the IT department is support from the relevant leadership of your department/college. Support must be provided not only in the form of finances, but also in administration and infrastructure.

Consider that decisions that are made for DP integration into a department's pre-existing organizational structure could impact aspects of teaching, research, and diagnostic services. The changes that could be required by the department to accommodate user abilities, the increase on the bandwidth at the institution, staff requirements for providing ready response to issues, etc. could be disruptive at best, but in some instances, transformational to the unit. Local level leadership should have input and buy-in at the outset to determine ultimate direction and footprint of the program, as well as how well (or not so well) it will fit into the current dogmas and schemas of the unit.

DP software and hardware are two costly non-IT related components. Although today's whole slide scanners are more affordable, they can still cost upwards of $\$ 100,000^{3}$. Additional "nice to have" capabilities that can be relevant for research but will add to the cost of the equipment include features such as fluorescence scanning capability (the number of channels that the machine is equipped with adds to cost), z-stacking, and, potentially, extended auto focus. The hardware and software will require annual/ongoing service and maintenance contracts and technical support as well.

Software to expand the pathologists' analytical capabilities (see below) can also be an expensive addition to the program. For complex image analyses there are several available software options and many seamlessly integrate with the lab's scanner systems. Image analysis software vendors sell a la carte services and/or tiered packages with varied pricing options. For example, the National Institutes of Health offer 2 free packages: Image J + the optional Fiji package; however, these programs are not as sophisticated as the paid programs $s^{4,5}$.
The analytical abilities among the commercial packages are similar (e.g. Visiopharm, Halo (Indica Labs), and Aiforia); however, the level of "supervision" of the programs that is required by the user, is often what differentiates the packages capabilities and costs ${ }^{6,7}$. The continued education and training of the DP team will be an additional expense that will need to be considered in the long-term cost structure.

A key addition to the workflow that is often overlooked in the beginning stages is an image management system that will allow for the delivery of the WSI to both internal and external customers. In most cases, IT is needed to help figure out the requirements that the system must have. For example, it would not be uncommon for one to need to use the same image management system to perform under different security levels:

1. An open access system that is not necessarily password protected to allow viewing of certain images, such as images used for tumor boards or resident rounds, simultaneously.

2. Delivering confidential research images both internally and externally

3. Allowing access to specific images to students within a certain class (the student list being confidential information) for a temporary time period.

The different levels of risk and regulation for each group is mind boggling to the average pathologist-hence the need for an IT professional that knows the lay of your land. Some scanners provide an image management system with the hardware and independent options (cost and capabilities vary considerably) exist that require an onsite data storage system at your institution, while others offer a paid cloud-based image system. Careful research of the capabilities of the system in the context of your primary use for the system will need to be done by the DP team leader with an IT professional's input.

\section{Analog to digital research pathology: A new mental model}

Digital pathology can be a highly impactful addition to the animal research histopathology workflow-greatly improving data delivery and access, as well as the efficiency and consistency of tissue analyses. Similar to the workflow in which only a light microscope is used, the process begins with quality tissue processing and the production of a glass slide. The process diverges after the glass slide is digitized with a whole slide scanner and a WSI is produced.

The most dramatic difference in the two processes can now be exploited - that is, the

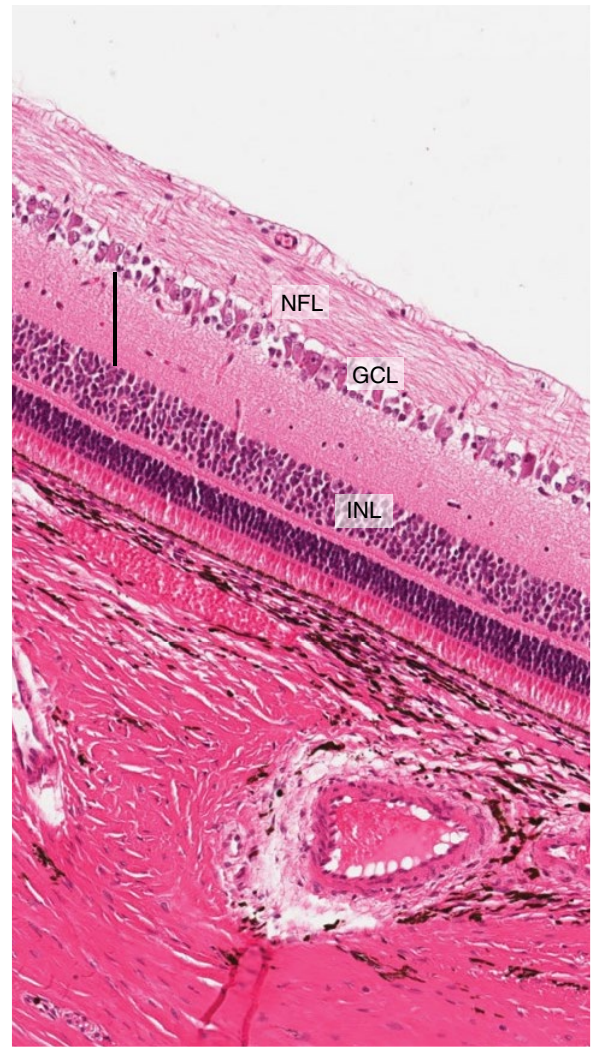

Fig. 1 | This example of computer assisted photography and morphometrical analysis is of a retina of a control pig from a chemically induced diabetic retinopathy model. The layers were identified for the researcher (nerve fiber layer, $\mathrm{NFL}$; ganglion cell layer, GCL; and inner nuclear layer; INL). Additionally, layers were measured (the bar is an example) in multiple places and averaged.

computer monitor as the virtual microscope (VM) to view the $\mathrm{WSI}^{8}$. The DP toolbox increases the pathologists' efficiency and productivity while advancing expanding their ability to analyze research samples. By adding DP to the workflow, the pathologist can easily perform simple, invaluable tasks such as annotating, photography and morphometry (Fig. 1), as well as more sophisticated analyses by using software to design programs/algorithms to annotate and analyze the WSI or regions of interest (ROI) in a section.

The application of artificial intelligence (AI), which includes deep learning and machine learning, to WSI can revolutionize data extraction and evaluation from, potentially, large data sets by enabling the pathologist to automate many analytical tasks (Fig. 2) and also perform higher level interrogation of sections (i.e. tissue segmentation, determination of cellularity, identification of metastases, etc.). The 


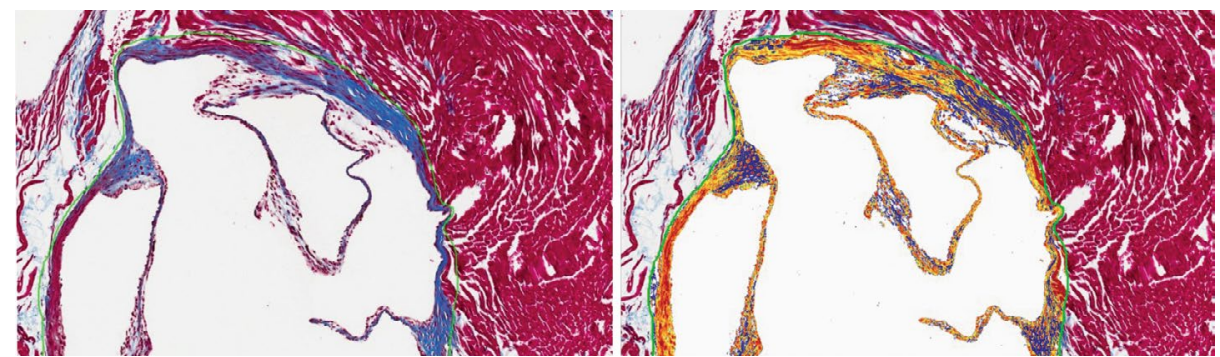

Fig. 2 | An algorithm was developed to detect fibrous tissue (blue) in the heart valves of trichrome stained sections (left). The algorithm was then run on batches of samples and the output was presented as percentages of the strength of positivity (intensity of blue staining). The numerical results could be exported to a spreadsheet and also could be visualized directly on the analyzed slide (right). Red, orange and yellow represent decreasing levels of intensity of positivity, respectively. Blue is a negative result, indicating on fibrosis detected.

outcome is faster availability of results that are objective rather than subjective and can be reported quantitatively rather than qualitatively ${ }^{9}$. For example, it could take the pathologist days to analyze and interpret hundreds of samples in a study via manual evaluation with a light microscope, and the output is a subjective, qualitive report. However, when AI enhanced software programs are used to complete analyses in large batches using algorithms, the output is an objective, reproducible and quantitative report that is available in hours.

Contrary to a prevailing concern by some, this technology will not supplant the pathologist, but instead will allow the pathologist to accomplish more, irrespective of location (as long as a computer and internet connection are accessible)making them more invaluable to research and available. To drive home the point, consider the fact that animal models are pivotal in drug discovery and development for both efficacy studies and drug safety assessments. Whole slide image analysis, deep learning and machine learning algorithms can be used on hematoxylin and eosin, immunohistochemistry and other stains to acquire objective measurements by automatically identifying features and/or ROI in tissue and categorize and differentiate individual cells or features. These data can be combined/correlated with expression levels of protein, RNA or DNA, the location of specific biomarkers and results from other bioinformatic analyses to strengthen and expand the conclusion derived from any given study $y^{6,10}$.
A recent review by the DPA outlines some considerations for integrating image analysis in a research setting ${ }^{10}$.

\section{Summary}

Digital pathology has the potential to transform research, teaching and diagnostics. Automated image analysis in research allows the veterinary pathologist to more quickly and efficiently produce quantitative, rather than descriptive, data on a larger scale and at a faster pace. Rest assured - the veterinary pathologist will not be cast aside, but will continue to serve as a valued research team member or leader. They will still be needed to assist in the experimental design, including advising on the most relevant animal model, ensuring that analyses are correctly performed and helping to determine what tests are best used to assess what and where pathology (or lack thereof) occurs in the study animals ${ }^{11-13}$.

However, to fully realize the advantages of DP one must obtain support and commitment to the program from the organizational leadership; have the initial and sustained integration of IT in the process; and get buy-in from the change agents amongst the pathology team. Some limitations of the DP workflow must also be kept in perspective, including: the need for standardization of the algorithms in order to improve reproducibility; inherent limitations of most hardware for processing and storing large files; the costs can be significant for starting and maintaining the program; image analysis software costs and training time should be considered; algorithms that have been validated in one lab, using a particular software program may not be reproducible in another lab for a variety of reasons, thus full protocols should be published to include vendors used and, if possible, algorithm details ${ }^{14,15}$. Lastly, a positive on the horizon is the current trend of teaching histopathology (in both veterinary and medical schools) using digitally scanned images of tissue sections. This shift has the field poised to embrace this technology wholeheartedly with the new generation of pathologists and will likely eliminate the hurdle of pathologists' resistance.

\section{Yava Jones-Hall ${ }^{凶}$}

Texas A\&M University, College Station, Texas, USA.

$\bigotimes_{e-m a i l: y a v a j h @ c v m . t a m u . e d u}$

Published online: 4 August 2021

https://doi.org/10.1038/s41684-021-00828-6

References

1. Zarella, M. D. et al. Arch Pathol Lab Med 143, 222-234, https://doi.org/10.5858/arpa.2018-0343-RA (2018).

2. Abels, E. et al. J Pathol 249, 286-294, https://doi.org/10.1002/ path.5331 (2019).

3. Cornish, T. C., Swapp, R. E. \& Kaplan, K. J. Adv Anat Pathol 19 152-159, https://doi.org/10.1097/PAP.0b013e318253459e (2012).

4. Schindelin, J. et al. Nat Method 9, 676-682, https://doi. org/10.1038/nmeth.2019 (2012).

5. Schneider, C. A., Rasband, W. S. \& Eliceiri, K. W. Nat Method 9 , 671-675, https://doi.org/10.1038/nmeth.2089 (2012).

6. Webster, J. D. \& Dunstan, R. W. Vet Pathol 51, 211-223, https://doi.org/10.1177/0300985813503570 (2014).

7. Saravanan, C. et al. Toxicol Pathol 45, 983-1003, https://doi.org/ 10.1177/0192623317737468 (2017).

8. Bertram, C. A. \& Klopfleisch, R. Vet Pathol 54, 756-766, https://doi.org/10.1177/0300985817709888 (2017).

9. Salto-Tellez, M., Maxwell, P. \& Hamilton, P. Histopathology 74 372-376, https://doi.org/10.1111/his.13760 (2019).

10. Aeffner, F. et al. J Pathol Inform 10, 9, https://doi.org/10.4103/ jpi.jpi_82_18 (2019).

11. Ferreira, G. S. et al. Plos One 14, e0218014, https://doi. org/10.1371/journal.pone.0218014. (2021).

12. Denayer, T., Stöhr, T. \& Van Roy, M. New Horiz Transl Med 2, 5-11, https://doi.org/10.1016/j.nhtm.2014.08.001 (2014).

13. Knoblaugh, S. E., Hohl, T. M. \& La Perle, K. M. D. Ilar J 59, 40-50, https://doi.org/10.1093/ilar/ilz001 (2018).

14. Acs, B. \& Hartman, J. J Pathol 250, 7-8, https://doi.org/10.1002/ path.5343 (2020).

15. Colling, R. et al. J Pathol 249, 143-150, https://doi.org/10.1002/ path.5310 (2019).

\section{Acknowledgements}

The author is grateful for the technical assistance and advice provided by Jeff Skelton, Senior Information Technology Manager at Texas A\&M (TAMU) College of Veterinary Medicine and Biomedical Science (CVMBS), which was instrumental to the successful implementation of our college's digital pathology program. Additionally, a heartfelt thanks is extended to Dr. Garry Adams, senior professor and anatomic pathologist at TAMU CVMBS for his insight, wisdom and support during the implementation of our college's DP program. 